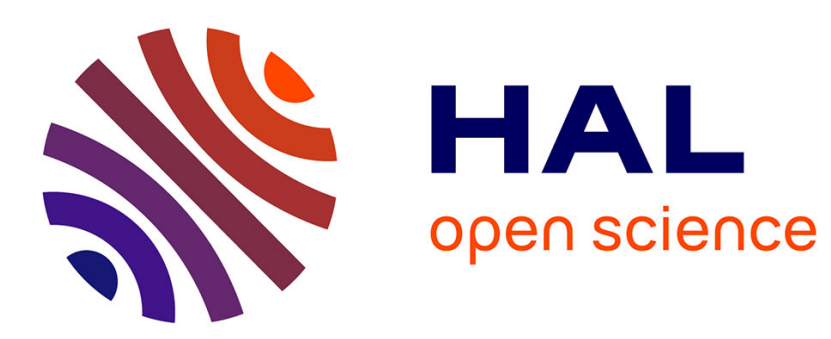

\title{
Isotropic Design of the Spherical Wrist of a Cable-Driven Parallel Robot
}

Angelos Platis, Tahir Rasheed, Philippe Cardou, Stéphane Caro

\section{To cite this version:}

Angelos Platis, Tahir Rasheed, Philippe Cardou, Stéphane Caro. Isotropic Design of the Spherical Wrist of a Cable-Driven Parallel Robot. Lenarcic J.; Merlet JP. Advances in Robot Kinematics 2016, 4, pp.321-330, 2017, Springer Proceedings in Advanced Robotics., 10.1007/978-3-319-56802-7_34 . hal-01758077

\section{HAL Id: hal-01758077 https://hal.science/hal-01758077}

Submitted on 4 Apr 2018

HAL is a multi-disciplinary open access archive for the deposit and dissemination of scientific research documents, whether they are published or not. The documents may come from teaching and research institutions in France or abroad, or from public or private research centers.
L'archive ouverte pluridisciplinaire HAL, est destinée au dépôt et à la diffusion de documents scientifiques de niveau recherche, publiés ou non, émanant des établissements d'enseignement et de recherche français ou étrangers, des laboratoires publics ou privés. 


\title{
Isotropic Design of the Spherical Wrist of a Cable-Driven Parallel Robot
}

Angelos Platis, Tahir Rasheed, Philippe Cardou, and Stéphane Caro

\begin{abstract}
Because of their mechanical properties, parallel mechanisms are most appropriate for large payload to weight ratio or high-speed tasks. Cable driven parallel robots (CDPRs) are designed to offer a large translation workspace, and can retain the other advantages of parallel mechanisms. One of the main drawbacks of CDPRs is their inability to reach wide ranges of end-effector orientations. In order to overcome this problem, we introduce a parallel spherical wrist (PSW) end-effector actuated by cable-driven omni-wheels. In this paper we mainly focus on the description of the proposed design and on the appropriate placement of the omni-wheels on the wrist to maximize the robot dexterity.
\end{abstract}

Key words: Parallel mechanism, cable-driven parallel robot, parallel spherical wrist, wrenches, dexterity

\section{Introduction}

Several applications could benefit from CDPRs endowed with large orientation workspaces, such as entertainment and manipulation and storage of large and heavy parts. This component of the workspace is relatively small in existing CDPR designs.To resolve this problem, a parallel spherical wrist (PSW) end-effector is introduced and connected in series with the translational 3-DOF CDPR to provide an unbounded singularity-free orientation workspace.

IRCCyN, École Centrale de Nantes, 1 rue de la Noë, 44321, Nantes, France, e-mail: \{Angelos.Platis, Tahir.Rasheed\}@eleves.ec-nantes.fr

Laboratoire de robotique, Département de génie mécanique, Université Laval, Quebec City, QC, Canada. e-mail: pcardou@gmc.ulaval.ca

CNRS-IRCCyN, 1 rue de la Noë, 44321, Nantes, France, e-mail: stephane.caro@irccyn.ecnantes.fr 
This paper focuses on the kinematic design and analyis of a PSW actuated by the cables of a CDPR providing the robot independent translation and orientation workspaces. CDPRs are generally capable of providing a large 3-dofs translation workspace, normally needed four cables, which enable the user to control the point where all of them are concentrated [2], [5].

Robots that can provide large orientation workspace have been developed using spherical wrist in the past few years that allows the end-effector to rotate with unlimited rolling, in addition to a limited pitch and yaw movements [3], [10]. Eclipse II [9] is an interesting robot that can provide unbounded 3-dofs translational motions, however its orientation workspace is constrained by structural interference and rotation limits of the spherical joints.

Several robots have been developed in the past having decoupled translation and rotational motions. One interesting concept of such a robot is that of the Atlas Motion Platform [6] developed for simulation applications. Another robot with translation motions decoupled from orientation motions can be found in [11]. The decoupled kinematics are obtained using a triple spherical joint in conjunction with a 3-UPS parallel robot.

In order to design a CDPR with a large orientation workspace, we introduce a parallel spherical wrist (PSW) end-effector actuated by cable-driven omni-wheels. In this paper we mainly focus on the description of the proposed design and on the appropriate placement of the omni-wheels on the wrist to maximize the robot dexterity.

\section{Manipulator Architecture}

The end-effector is a sphere supported by actuated omni-wheels as shown in Fig. 1. The wrist contians three passive ball joints at the bottom and three active omniwheels being driven through drums. Each cable makes several loops around each drum. Both ends are connected to two servo-actuated winches, which are fixed to the base. When two servo-actuated winches connected to the same cable turn in the same direction, the cable circulates and drives the drum and its associated omniwheel. When both servo-actuated winches turn in opposite directions, the length of the cable loop changes, and the sphere centre moves. To increase the translation workspace of the CDPR, another cable is attached, which has no participation in the omni-wheels rotation. The overall design of the manipulator is shown in Fig. 2.

We have in total three frames. First, the CDPR base frame $\left(\mathscr{F}_{0}\right)$, which is described by its center $O_{0}$ having coordinates $x_{0}, y_{0}, z_{0}$. Second, the PSW base frame $\left(\mathscr{F}_{1}\right)$, which has its center $O_{1}$ at the geometric center of the sphere and has coordinates $x_{1}, y_{1}, z_{1}$. Third, the spherical end-effector frame $\left(\mathscr{F}_{2}\right)$ is attached to the end-effector. Its centre $\mathrm{O}_{2}$ coincides with that of the PSW base frame $\left(\mathrm{O}_{2} \equiv \mathrm{O}_{1}\right)$ and its coordinates are $x_{2}, y_{2}, z_{2}$.

Exit points $A_{i}$ are the cable attachment points that link the cables to the base. All exit points are fixed and expressed in the CDPR reference frame $\mathscr{F}_{0}$. Anchor points $B_{i}$ are the platform attachment points. These points are not fixed as they depend 


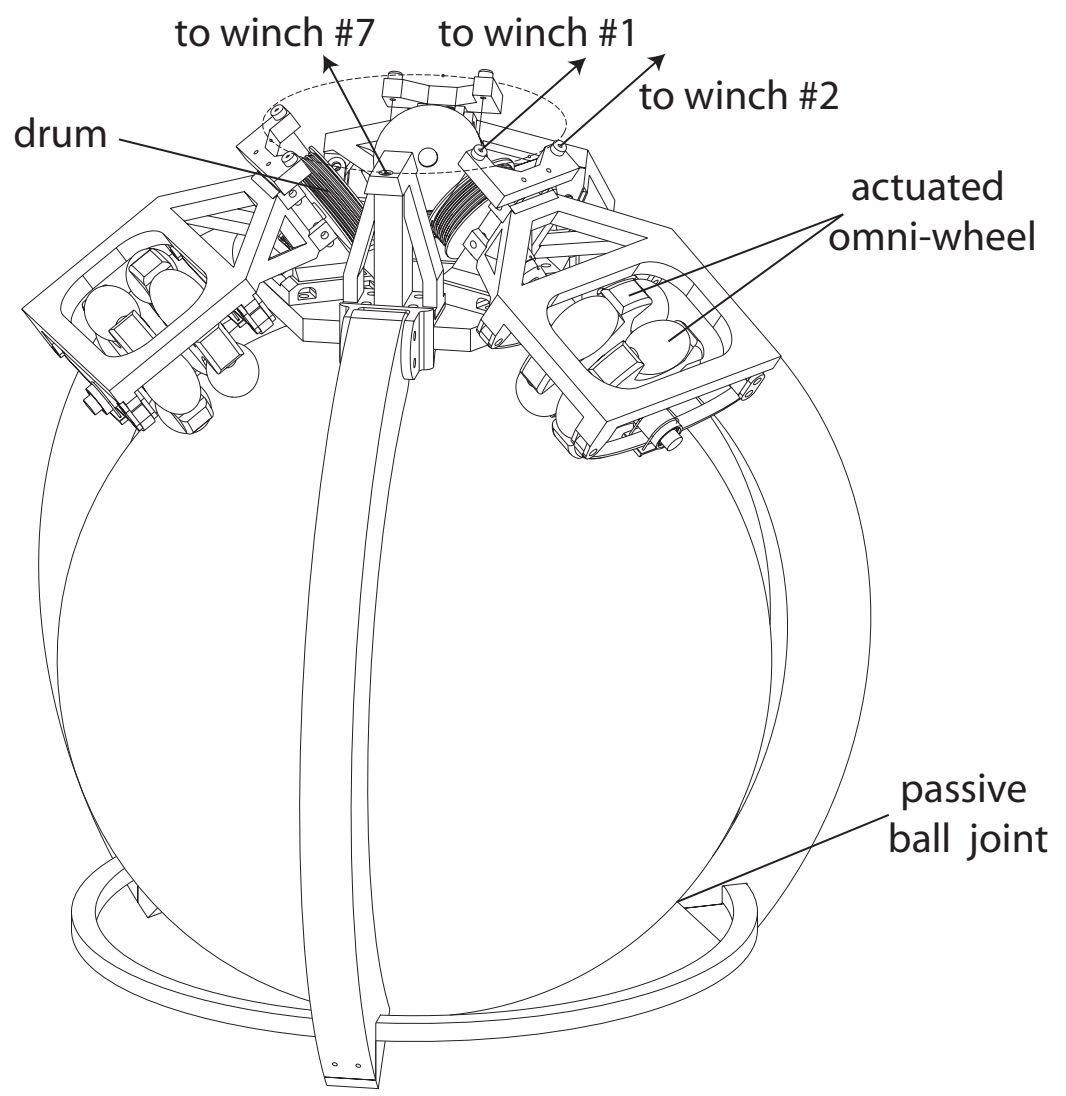

Fig. 1: Isotropic design of the parallel spherical wrist

on the vector $P$, which is the vector that contains the pose of the moving platform expressed in the CDPR reference frame $\mathscr{F}_{0}$. The remaining part of the paper aims at finding the appropriate placement of the omni-wheels on the wrist to maximise the robot dexterity.

\section{Kinematic Analysis of the Parallel Spherical Wrist}

\subsection{Parameterization}

To simplify the parameterization of the parallel spherical wrist, some assumptions are made. First, all the omni-wheels are supposed to be normal the sphere. Second, the contact points of the omni-wheels with the sphere lie in the base of an inverted cone where its end is the geometrical center of the sphere parametrized by angle $\alpha$. 


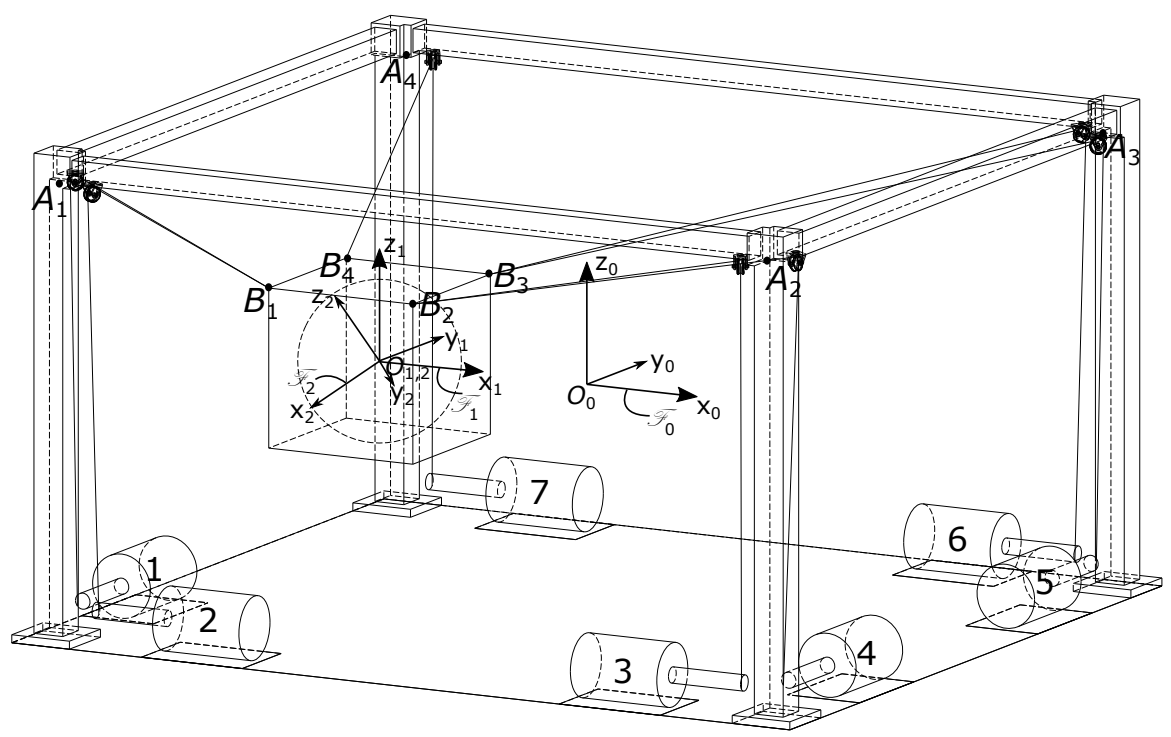

Fig. 2: Concept idea of the manipulator

Third, the three contact points form an equilateral triangle as shown in $[6,7]$. Fourth, the angle between the tangent to the sphere and the actuation force produced by the $i$ th actuated omni-wheel is named $\beta_{i}, i=1,2,3$, and $\beta_{1}=\beta_{2}=\beta_{3}=\beta$. Figure 3 illustrates the sphere, one actuated omni-wheel and the main design variables of the parallel spherical wrist. $\Pi_{i}$ is the plane tangent to the sphere and passing through the contact point $G_{i}$ between the actuated omni-wheel and the sphere.

$\omega_{i}$ denotes the angular velocity vector of the $i$ th actuated omni-wheel. $\mathbf{s}_{i}$ is a unit vector along the tangent line $\mathcal{T}$ that is tangent to the base of the cone and coplanar to plane $\Pi_{i} . \mathbf{w}_{i}$ is a unit vector normal to $\mathbf{s}_{i} . \mathbf{f}_{a i}$ depicts the transmission force lying in plane $\Pi_{i}$ due to the actuated omni-wheel. $\alpha$ is the angle defining the altitude of contact points $G_{i}(\alpha \in[0, \pi])$. $\beta$ is the angle between the unit vectors $\mathbf{s}_{i}$ and $\mathbf{v}_{i}$ $\left(\beta \in\left[-\frac{\Pi}{2}, \frac{\Pi}{2}\right]\right)$. As the contact points $G_{i}$ are the corners of an equilateral triangle, the angle between the contact point $G_{1}$ and the contact points $G_{2}$ and $G_{3}$ is equal to $\gamma . R$ is the radius of the sphere. $r_{i}$ is radius of the $i^{t h}$ actuated omni-wheel. $\dot{\varphi}_{i}$ is the angular velocity of the omni-wheel. $\mathbf{u}_{i}, \mathbf{v}_{i}, \mathbf{n}_{i}$ are unit vectors at point $G_{i}$ and $\mathbf{i}, \mathbf{j}, \mathbf{k}$ are unit vectors along $x_{2}, y_{2}, z_{2}$ respectively.

In order to analyze the kinematic performance of the parallel spherical wrist, an equivalent parallel robot (Fig. 4) having six virtual legs is presented, each leg having a spherical, a prismatic and another spherical joints connected in series. Three legs have an actuated prismatic joint (green), whereas the other three legs have a locked prismatic joints (red). Here, the kinematics of the spherical wrist is analyzed with screw theory and an equivalent parallel robot represented in Fig. 4. 


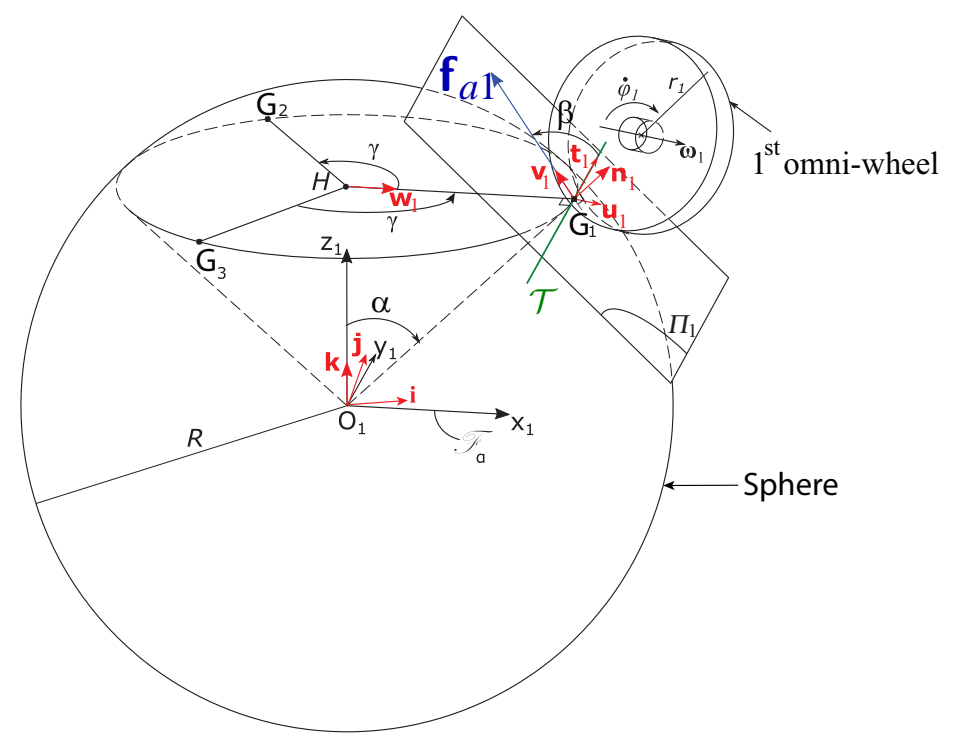

Fig. 3: Parameterization of the parallel spherical wrist

\subsection{Kinematic Modeling}

Fig. 4(a) represents the three actuation forces $\mathbf{f}_{a i}, i=1,2,3$ and the three constraint forces $\mathbf{f}_{c i}, i=1,2,3$ exerted by the actuated omni-wheels on the sphere. The three constraint forces intersect at the geometric center of the sphere and prevent the latter from translating. The three actuation forces generated by the three actuated omniwheels allow us to control the three-dof rotational motions of the sphere. Fig. 4(b) depicts a virtual leg corresponding to the effect of the $i$ th actuated omni-wheel on the sphere. The kinematic model of the PSW is obtained by using the theory of reciprocal screws $[4,8]$ as follows:

$$
\mathbf{A} \mathbf{t}=\mathbf{B} \dot{\varphi}
$$

where $\mathbf{t}$ is the sphere twist, $\dot{\varphi}=\left[\begin{array}{lll}\dot{\varphi}_{1} & \dot{\varphi}_{2} & \dot{\varphi}_{3}\end{array}\right]^{T}$ is the actuated omni-wheel angular velocity vector. A and $\mathbf{B}$ are respectively the forward and inverse kinematic Jacobian matrices of the PSW and take the form:

$$
\begin{aligned}
& \mathbf{A}=\left[\begin{array}{cc}
\mathbf{A}_{r \omega} & \mathbf{A}_{r p} \\
\mathbf{0}_{3 \times \mathbf{3}} & \mathbf{I}_{3}
\end{array}\right] \\
& \mathbf{B}=\left[\begin{array}{c}
\mathbf{I}_{3} \\
\mathbf{0}_{3 \times 3}
\end{array}\right]
\end{aligned}
$$



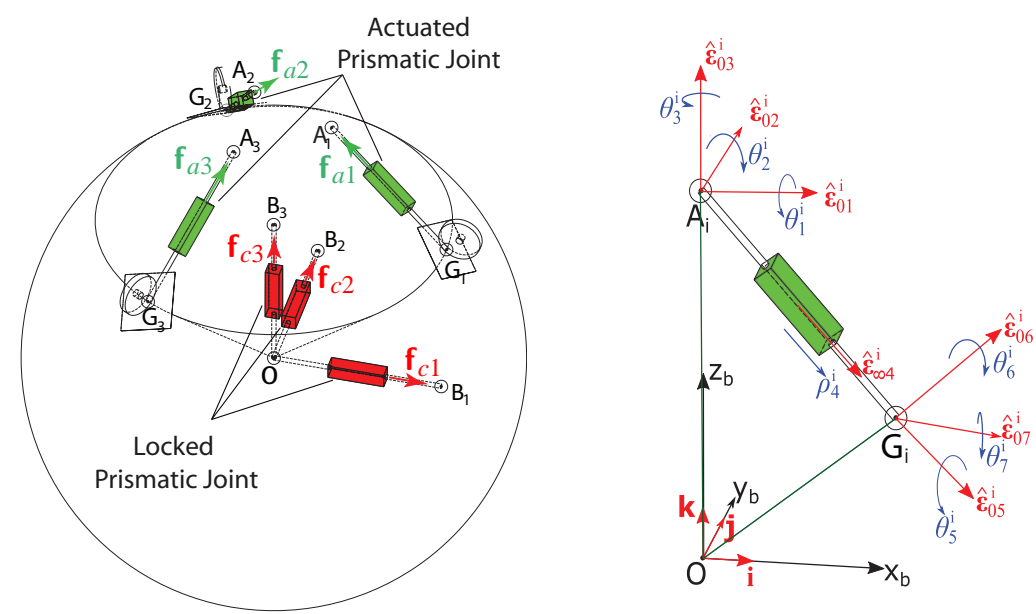

Fig. 4: (a) Actuation and constraint wrenches applied on the end-effector of the spherical wrist (b) Virtual $i^{\text {th }}$ leg with actuated prismatic joint

$\mathbf{I}_{3}$ and $\mathbf{0}_{3 \times 3}$ are the $3 \times 3$ identity and zeros matrices, and the matrices $\mathbf{A}_{r \omega}$ and $\mathbf{A}_{r p}$ are:

$$
\mathbf{A}_{r \omega}=\left[\begin{array}{c}
R\left(\mathbf{n}_{1} \times \mathbf{v}_{1}\right)^{T} \\
R\left(\mathbf{n}_{2} \times \mathbf{v}_{2}\right)^{T} \\
R\left(\mathbf{n}_{3} \times \mathbf{v}_{3}\right)^{T}
\end{array}\right] \text { and } \mathbf{A}_{r p}=\left[\begin{array}{c}
\mathbf{v}_{1}^{T} \\
\mathbf{v}_{2}^{T} \\
\mathbf{v}_{3}^{T}
\end{array}\right]
$$

As the contact points on the sphere form an equilateral triangle, $\gamma=2 \pi / 3$. As a consequence, matrices $\mathbf{A}_{r \omega}$ and $\mathbf{A}_{r p}$ are expressed as functions of the design parameters $\alpha$ and $\beta$ :

$$
\begin{aligned}
& \mathbf{A}_{r \omega}=\frac{R}{2}\left[\begin{array}{ccc}
-2 C \alpha C \beta & -2 S \beta & 2 S \alpha C \beta \\
C \alpha C \beta+\sqrt{3} S \beta & S \beta-\sqrt{3} C \alpha C \beta & 2 S \alpha C \beta \\
C \alpha C \beta-\sqrt{3} S \beta & S \beta+\sqrt{3} C \alpha C \beta & 2 S \alpha C \beta
\end{array}\right] \\
& \mathbf{A}_{r p}=\frac{1}{2}\left[\begin{array}{ccc}
-2 C \alpha S \beta & 2 C \beta & 2 S \alpha S \beta \\
C \alpha S \beta-\sqrt{3} C \beta & -(\sqrt{3} C \alpha S \beta+C \beta) & 2 S \alpha S \beta \\
C \alpha S \beta+\sqrt{3} C \beta & \sqrt{3} C \alpha S \beta-C \beta & 2 S \alpha S \beta
\end{array}\right]
\end{aligned}
$$

where $C$ and $S$ denote the cosine and sine functions, respectively.

\subsection{Singularity Analysis}

As matrix $\mathbf{B}$ cannot be rank deficient, the parallel spherical wrist meets singularities if and only if (iff) matrix $\mathbf{A}$ is singular. From Eqs. (5) and (6), matrix $\mathbf{A}$ is singular 


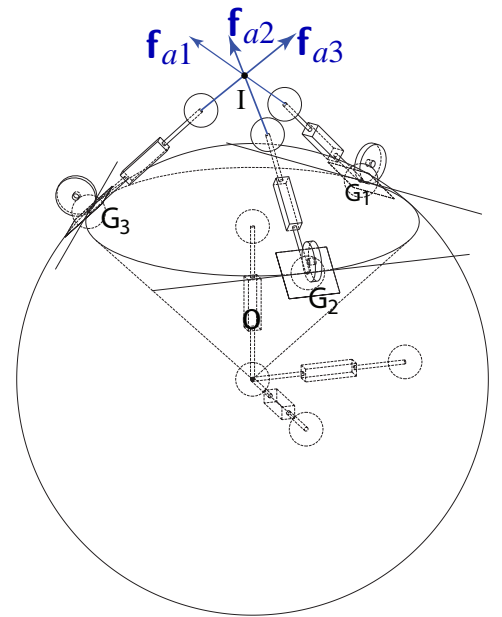

(a) $\beta= \pm \pi / 2$

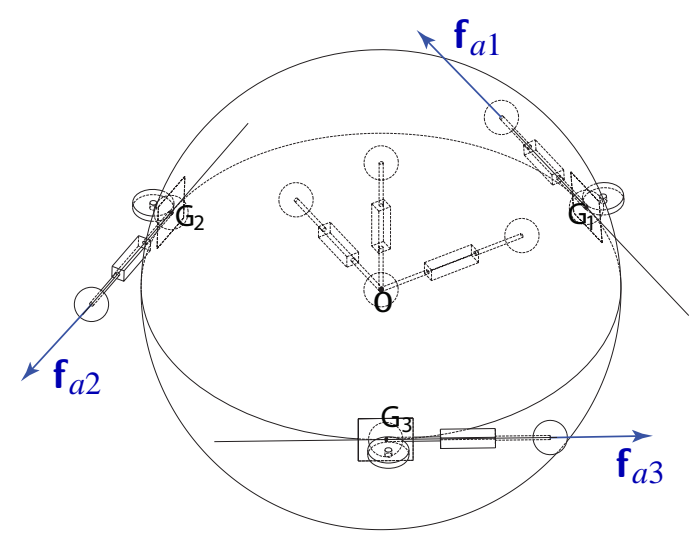

(b) $\alpha=\pi / 2$ and $\beta=0$

Fig. 5: Singular configurations of the parallel spherical wrist

iff:

$$
\operatorname{det}(\mathbf{A})=\frac{3 \sqrt{3}}{2} R^{3} S \alpha C \beta\left(1-S^{2} \alpha C^{2} \beta\right)=0
$$

namely, if $\alpha=0$ or $\pi$; if $\beta= \pm \pi / 2$; if $\alpha=\pi / 2$ and $\beta=0$ or $\pm \pi$.

Figs. 5a and 5b represent two singular configurations of the parallel spherical wrist under study. The three actuation forces $\mathbf{f}_{a 1}, \mathbf{f}_{a 2}$ and $\mathbf{f}_{a 3}$ intersect at point $I$ in Fig. 5a. The PSW reaches a parallel singularity and gains an infinitesimal rotation (uncontrolled motion) about an axis passing through points $O$ and $I$ in such a configuration. The three actuation forces $\mathbf{f}_{a 1}, \mathbf{f}_{a 2}$ and $\mathbf{f}_{a 3}$ are coplanar with plane $\left(X_{1} O Y_{1}\right)$ in Fig. 5b. The PSW reaches a parallel singularity and gains two-dof infinitesimal rotations (uncontrolled motions) about an axes that are coplanar with plane $\left(\mathrm{X}_{1} O Y_{1}\right)$ in such a configuration.

\subsection{Kinematically Isotropic Wheel Configurations}

This section aims at finding a good placement of the actuated omni-wheels on the sphere with regard to the manipulator dexterity. The latter is evaluated by the condition number of reduced Jacobian matrix $\mathbf{J}_{\omega}=r \mathbf{A}_{r \omega}^{-1}$ which maps angular velocities of the omni-wheels $\dot{\varphi}$ to the required angular velocity of the end-effector $\omega$. From Eqs. (5) and (6), the condition number $\kappa_{F}(\alpha, \beta)$ of $\mathbf{J}_{\omega}$ based on the Frobenius norm [1] is expressed as follows:

$$
\kappa_{F}(\alpha, \beta)=\frac{1}{3} \sqrt{\frac{3 S^{2} \alpha C^{2} \beta+1}{S^{2} \alpha C^{2} \beta\left(1-S^{2} \alpha C^{2} \beta\right)}}
$$




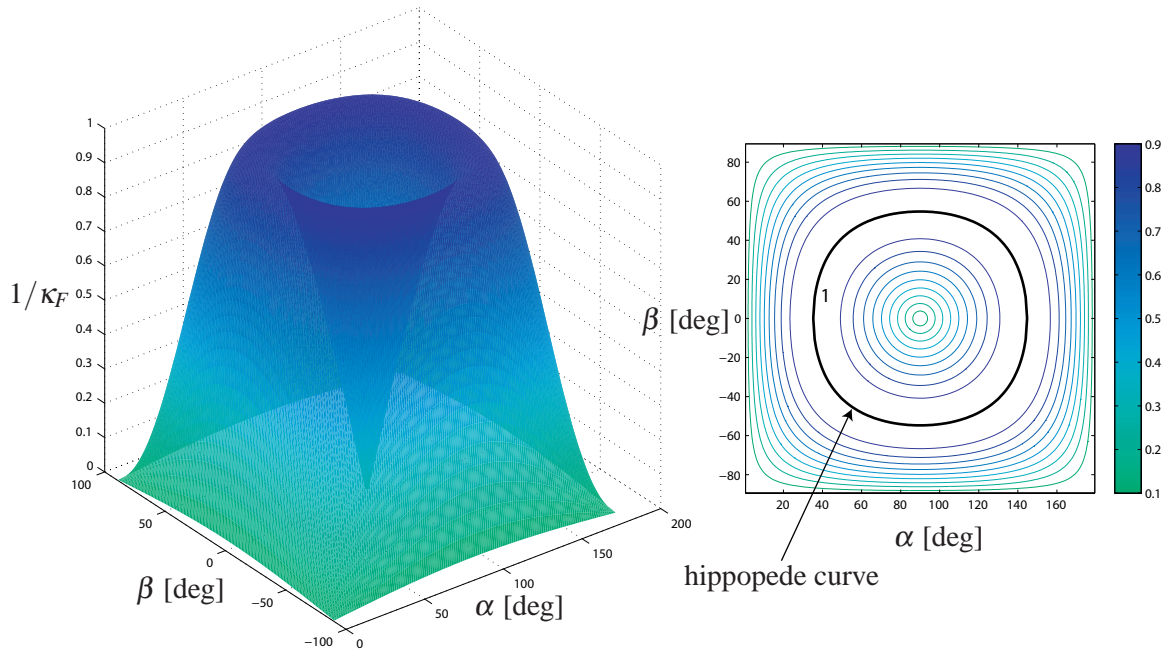

Fig. 6: Inverse condition number of the forward Jacobian matrix $\mathbf{A}$ based on the Frobenius norm as a function of design parameters $\alpha$ and $\beta$

Figure 6 depicts the inverse condition number of matrix A based on the Frobenius norm as a function of angles $\alpha$ and $\beta . \kappa_{F}(\alpha, \beta)$ is a minimum when its partial derivatives with respect to $\alpha$ and $\beta$ vanish, namely,

$$
\begin{aligned}
& \dot{\kappa}_{\alpha}(\alpha, \beta)=\frac{\partial \kappa}{\partial \alpha}=\frac{C \alpha\left(3 S^{2} \alpha C^{2} \beta-1\right)\left(S^{2} \alpha C^{2} \beta+1\right)}{18 S^{3} \alpha C^{2} \beta\left(S^{2} \alpha C^{2} \beta-1\right)^{2} \kappa}=0 \\
& \dot{\kappa}_{\beta}(\alpha, \beta)=\frac{\partial \kappa}{\partial \beta}=-\frac{S \beta\left(3 S^{2} \alpha C^{2} \beta-1\right)\left(S^{2} \alpha C^{2} \beta+1\right)}{18 S^{2} \alpha C^{3} \beta\left(S^{2} \alpha C^{2} \beta-1\right)^{2} \kappa}=0
\end{aligned}
$$

and its Hessian matrix is semi-positive definite. As a result, $\kappa_{F}(\alpha, \beta)$ is a minimum and equal to 1 along the hippopede curve, which is shown in Fig. 6 and defined by the following equation:

$$
3 S^{2} \alpha C^{2} \beta-1=0
$$

This hippopede curve amounts to the isotropic loci of the parallel spherical wrist.

Figure 7 illustrates some placements of the actuated omni-wheels on the sphere leading to kinematically isotropic wheel configurations in the parallel spherical wrist. It should be noted that the three singular values of matrix $\mathbf{A}_{r \omega}$ are equal to the ratio between the sphere radius $R$ and the actuated omni-wheel radius $r$ along the hippopede curve, namely, the velocity amplification factors of the PSW are the same and constant along the hippopede curve.

If the rotating sphere were to carry a camera, a laser or a jet of some sort, then the reachable orientations would be limited by interferences with the omni-wheels. 


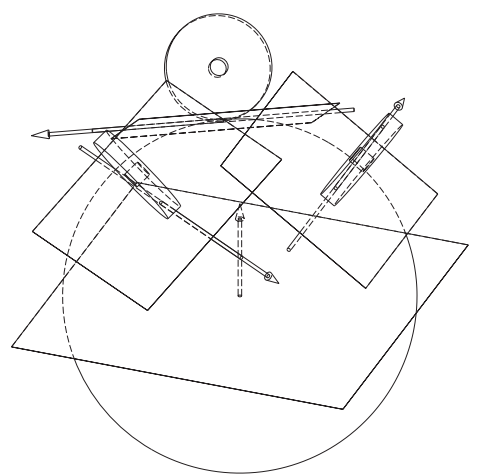

$\alpha=35.26^{\circ}, \beta=0^{\circ}$

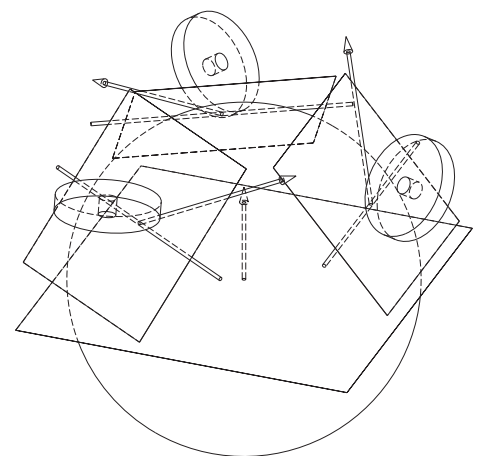

$\alpha=50^{\circ}, \beta=41.1^{\circ}$

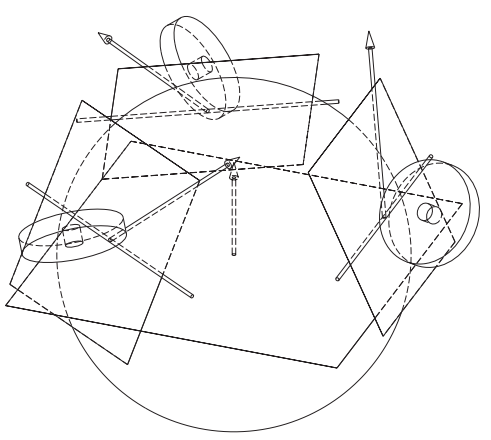

$\alpha=65^{\circ}, \beta=50.43^{\circ}$

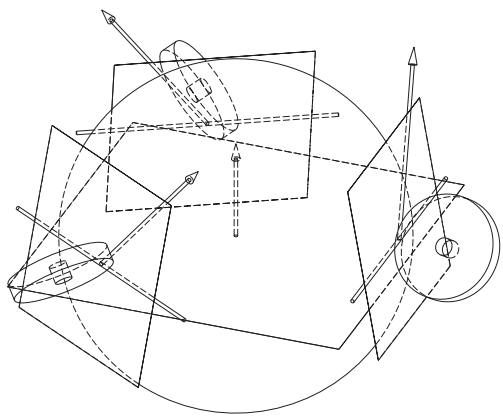

$\alpha=80^{\circ}, \beta=54.11^{\circ}$

Fig. 7: Kinematically isotropic wheel configurations in the parallel spherical wrist

Therefore, a designer would be interested in choosing a small value of alpha, so as to maximize the field of view of the PSW. As a result, the following values have been assigned to the design parameters $\alpha$ and $\beta$ :

$$
\begin{aligned}
& \alpha=35.26^{\circ} \\
& \beta=0^{\circ}
\end{aligned}
$$

in order to come up with a kinematically isotropic wheel configuration in the parallel spherical wrist and a large field of view. The actuated omni-wheels are mounted in pairs in order to ensure a good contact between them and the sphere. A CAD modeling of the final solution is represented in Fig. 1. 


\section{Conclusion}

This paper presents the novel concept of mounting a parallel spherical wrist in series with a CDPR, while preserving a fully-parallel actuation scheme. As a result, the actuators always remain fixed to the base, thus avoiding the need to carry electric power to the end-effector and minimizing its size, weight and inertia. Another original contribution of this article is the determination of the kinematically isotropic wheel configurations in the parallel spherical wrist. These configurations allow the designer to obtain a very good primary image of the design choices. To our knowledge, these isotropic configurations were never reported before, although several researchers have studied and used omni-wheel-actuated spheres. Future work includes the development of a control scheme to drive the end-effector rotations while accounting for the displacements of its centre, and also making a small scale prototype of the robot.

\section{References}

1. Angeles, J.: Fundamentals of Robotic Mechanical Systems: Theory, Methods and Algorithms, 3 edn. Springer-Verlag (2007)

2. Bahrami, A., Bahrami, M.N.: Optimal design of a spatial four cable driven parallel manipulator. Robotics and Biomimetics (ROBIO), 2011 IEEE International Conference pp. 2143 $2149(2011)$

3. Bai, S., Hansen, M.R.: Modelling of a spherical robotic wrist with euler parameters. 12th IFToMM World Congress (2007)

4. Ball, R.S.: A treatise on the theory of screws. Cambridge University Press,Cambridge,CA 23(41) (1900)

5. Hadian, H., Fattah, A.: Kinematic isotropic configuration of spatial cable-driven parallel robots. International Journal of Intelligent Mechatronics and Robotics 1(4), 61-86 (2011)

6. Hayes, M.J.D., Langlois, R.G.: Atlas motion platform: Full-scale prototype. In: CSME International Congress (2012)

7. Hayes, M.J.D., Langlois, R.G., Weiss, A.: Atlas motion platform generalized kinematic model. In: Meccanica, vol. 46, pp. 17-25 (2011)

8. Hunt, K.H.: Kinematic geometry of mechanisms. Clarendon Press, Oxford 23(41) (1978)

9. Kim, J., Hwang, J.C., Kim, J.S., Park, R.C.: Eclipse-ii: a new parallel mechanism enabling continuous 360-degree spinning plus three-axis translational motions. Robotics and Automation 4, $3274-3279$ (2001)

10. Wu, G., Caro, S., Bai, S., Kepler, J.: Dynamic modeling and design optimization of a 3-dof spherical parallel manipulator. Robotics and Autonomous Systems 62, 1377-1386 (2014)

11. Yime, E., Moreno, H., Saltarén, R.: A novel 6-dof parallel robot with decoupled translation and rotation. 13th World Congress in Mechanism and Machine Science (2011) 